\title{
Long-term rewritable report and recording of environmental stimuli in engineered bacterial populations
}

\author{
Zhen-Ping Zou ${ }^{1}$ and Bang-Ce Ye ${ }^{1,2 *}$ \\ ${ }^{1}$ Laboratory of Biosystems and Microanalysis, Institute of Engineering Biology and Health, State Key Laboratory of \\ Bioreactor Engineering, East China University of Science and Technology, Shanghai, 200237, China \\ ${ }^{2}$ Institute of Engineering Biology and Health, Collaborative Innovation Center of Yangtze River Delta Region Green \\ Pharmaceuticals, College of Pharmaceutical Sciences, Zhejiang University of Technology, Hangzhou 310014 , \\ Zhejiang, China
}




\section{Supplementary Figures}

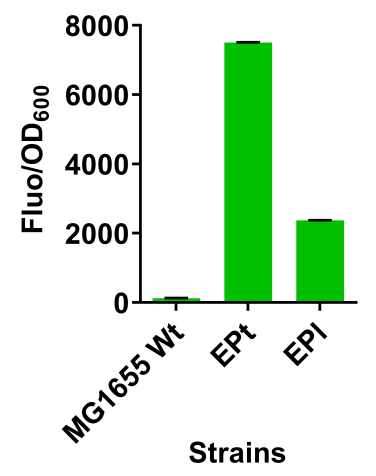

Figure S1. Fluorescence intensity of EPt and EPl. sfGFP was drivened by promoters (Ptac and Plac) in E.coil. Values and error bars are shown as mean $\pm \operatorname{SD}(n=3)$. 


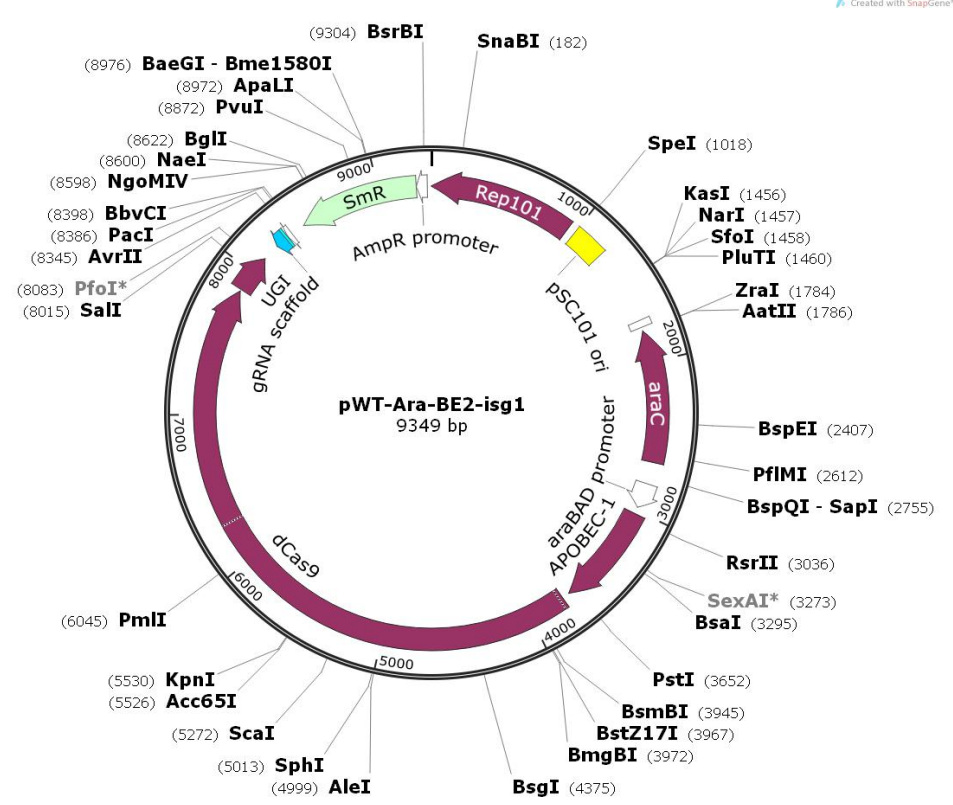

Figure S2. Detail profiles of the writing plasmid pWT-Ara-BE2-isg1

\section{$>$ Pj23119}

ttgacagctagctcagtcctaggtataatgctagc

>isgRNA1, sgRNA scaffold

catccagttccaccagaatagttttagagctagaaatagcaagttaaaataaggctagtccgttatcaacttgaaaaagtggcaccgagtcggtgctttttt

\section{>Arabinose-inducible promoter}

aaagccatgacaaaaacgcgtaacaaaagtgtctataatcacggcagaaaagtccacattgattatttgcacggcgtcacactttgctatgccatagcatttttatccataagat tagcggatcctacctgacgcttttatcgcaactctctactgtttctccat 


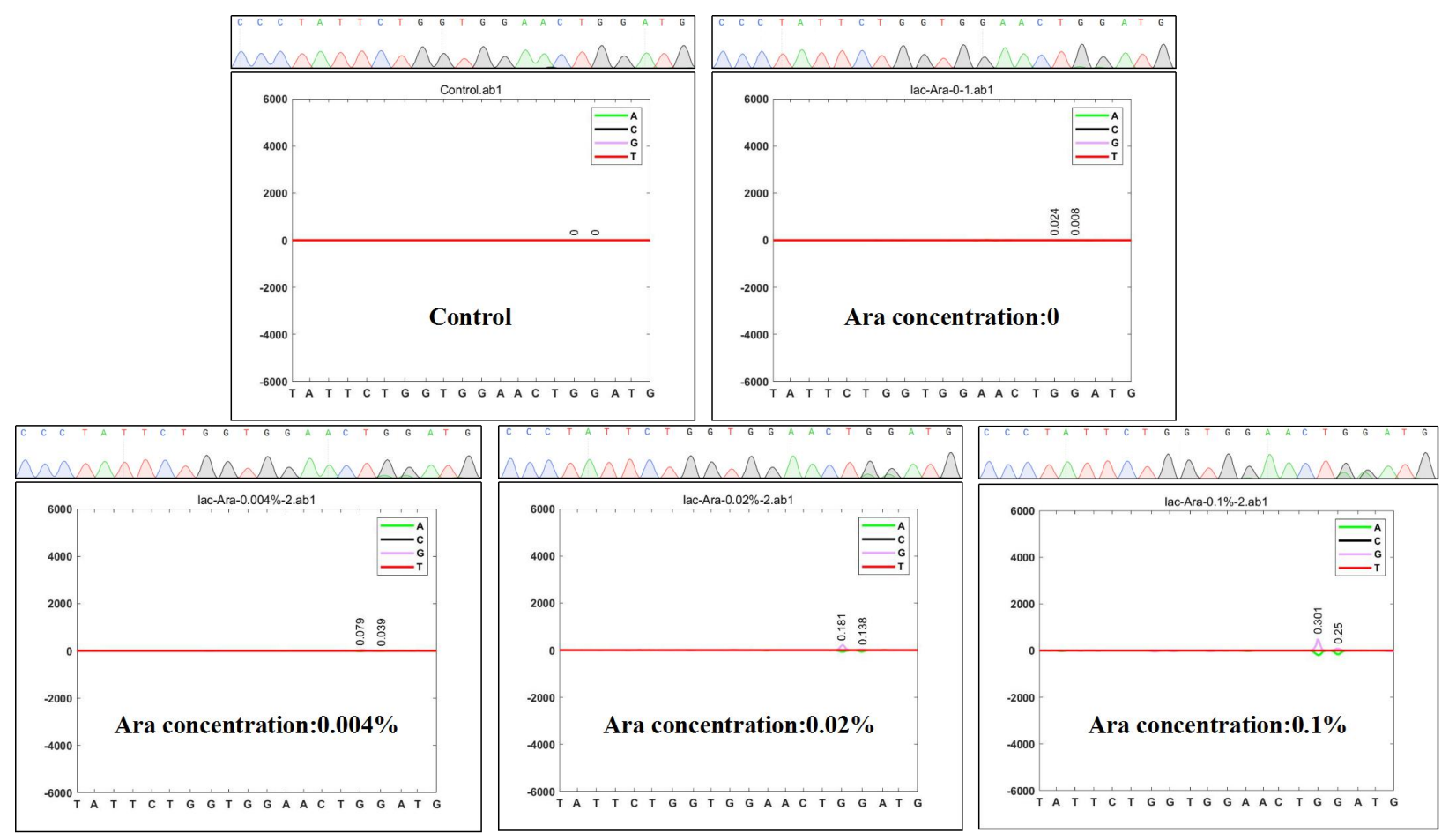

Figure S3. Sequalizer analysis. Sequalizer analyzes the frequency of base mutations at specific sites at different Ara concentrations. Software: MATLAB R2018a 


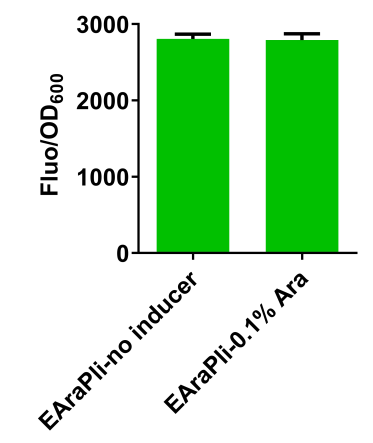

Figure S4. Fluorescence intensity of induced and uninduced bacteria after removal of inducer. EAraPli with or without $0.1 \%$ arabinose was activated overnight under the condition of no inducer, and then diluted the culture 200 times $(\mathrm{v} / \mathrm{v})$ to $5 \mathrm{~mL}$ of fresh LB culture $12 \mathrm{~h}$ and then the bacterial fluorescence intensity was measured. Values and error bars are shown as mean $\pm \mathrm{SD}(\mathrm{n}=3)$. 
A

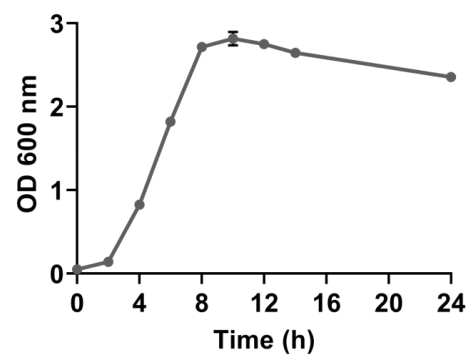

B

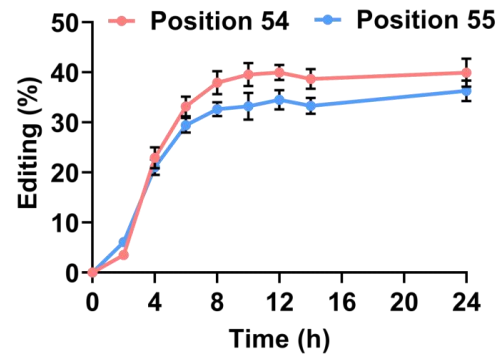

Figure S5. Dynamics of base mutations accumulation in EAraPli. (A) Growth curves of EAraPli of exposure to the $0.1 \%$ Ara. (B) Mutation accumulation of EAraPli strain. The culture was grown in a $30 \mathrm{~mL}$ shake flask with shaking, and $200 \mu \mathrm{L}$ of the sample was transferred to a 96-well transparent plate at the designed time point to measure the absorbance at $600 \mathrm{~nm}$, and then the base edit rate was determined. Values and error bars are shown as mean $\pm \mathrm{SD}$ $(n=3)$. 


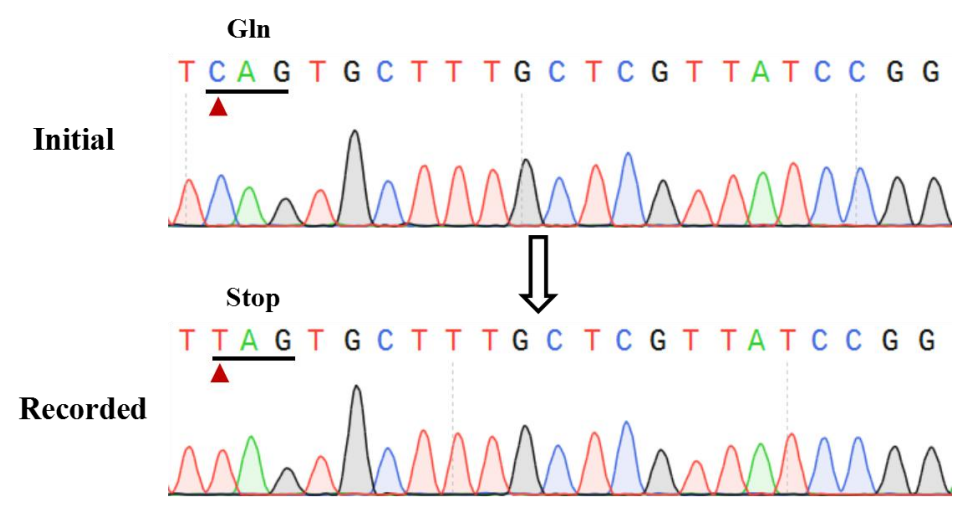

Figure S6. Theoretical unmutated and mutant site sanger sequencing chromatograms. The 205 base of the sfGFP of the initial strain is $\mathrm{C}$ and the mutanted (recorded) strain is $\mathrm{T}$. 


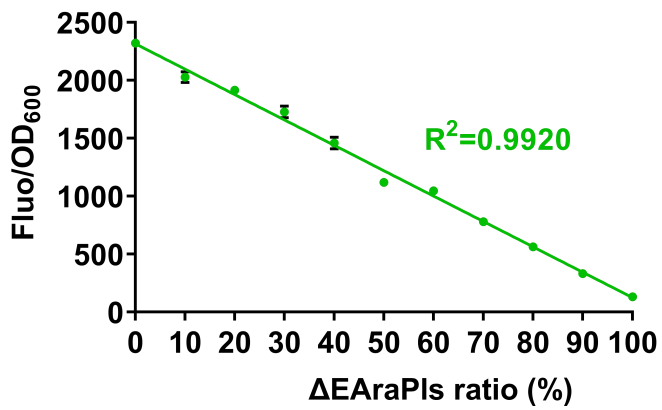

Figure S7. Standard curve obtained from samples with known mutant ratios by fluorescence intensity. The fluorescence intensity is linearly related to the ratio of the two strains, so we can estimate the bacterial ratio (base edit rate) by the change in the fluorescence value of the bacterial population. Values and error bars are shown as mean \pm $\mathrm{SD}(\mathrm{n}=3)$. 


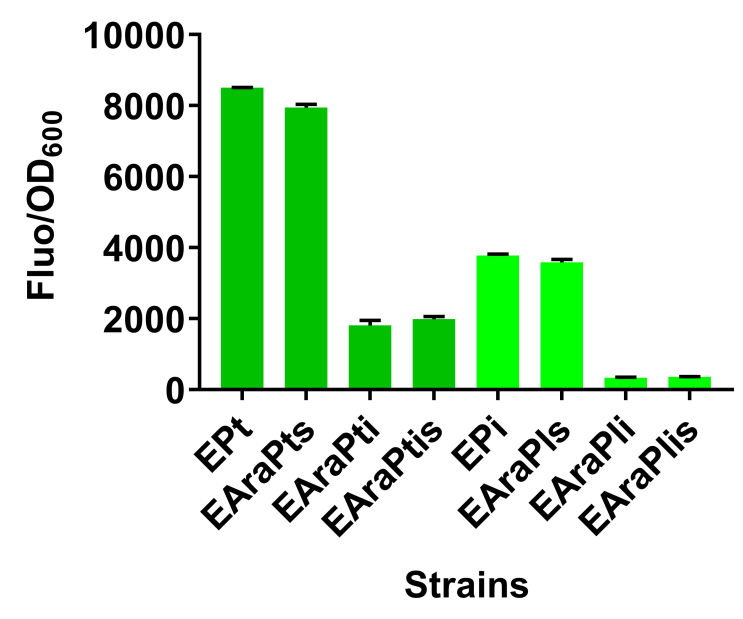

Figure S8. Fluorescence intensity of different strains under $0.1 \%$ Ara. After 12 hours of $0.1 \%$ Ara induction, the fluorescence intensity of strains carrying different systems were measured, the fluorescence intensity of strains carrying CRISPR-i (EAraPti, EAraPtis, EAraPli and EAraPlis) reduced significantly, and the fluorescence intensity of strains only carrying CRISPR-stop (EAraPts and EAraPls) reduced slightly. Values and error bars are shown as mean $\pm \mathrm{SD}(\mathrm{n}=3)$. 


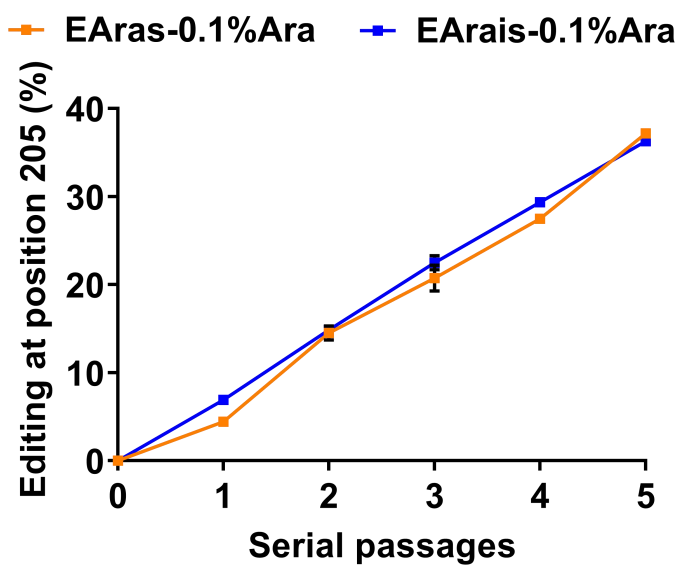

Figure S9. Recording of the stimuli duration using in EAras and EArais. Continuous determination of the editing effect of the CRISPR-stop and CRISPR-istop system on promoter-free upstream GFP expression cassette at position 205. Values and error bars are shown as mean $\pm \operatorname{SD}(n=3)$. 


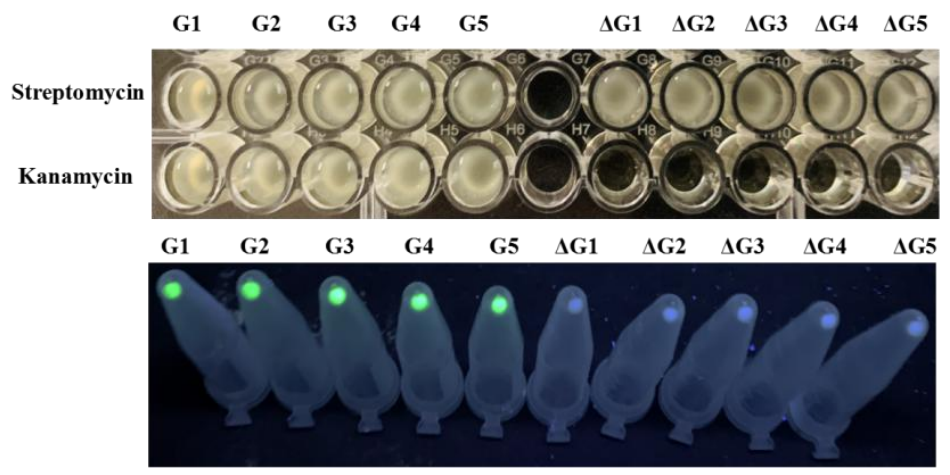

Figure S10. Fluorescence expression and resistance assay confirm functional coupling of sfGFP protein to kanamycin resistance protein. The unmutated strain had both fluorescent expression and kanamycin resistance, while the strain containing the 205 base mutation of sfGFP lost both functions. G1-5: strains cotains pWT-msfGFP-kanR; $\Delta$ G1-5: strains cotains $\mathrm{pWT}-\mathrm{m} \Delta \mathrm{sfGFP}-\mathrm{kanR}$. 
A

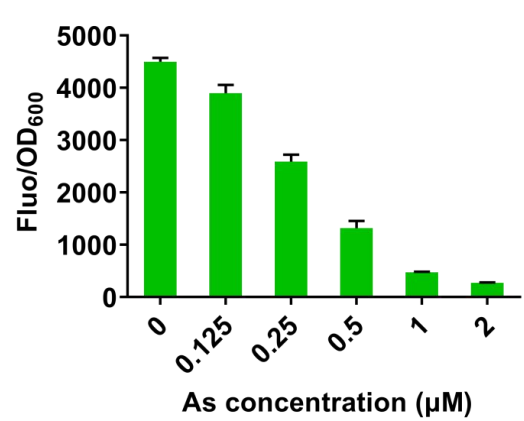

B

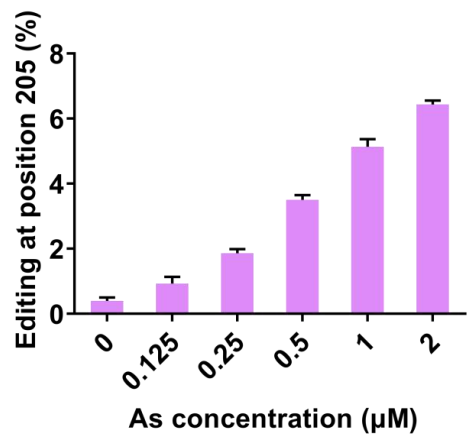

Figure S11. The detection range of the arsenic sensor with CRISPR-istop v2 system. sfGFP gene expression intensity changes (left) and the base editing rate of the sfGFP gene at position 205 (right) with As at different concentrations. Values and error bars are shown as mean $\pm \mathrm{SD}(\mathrm{n}=3)$. 
A
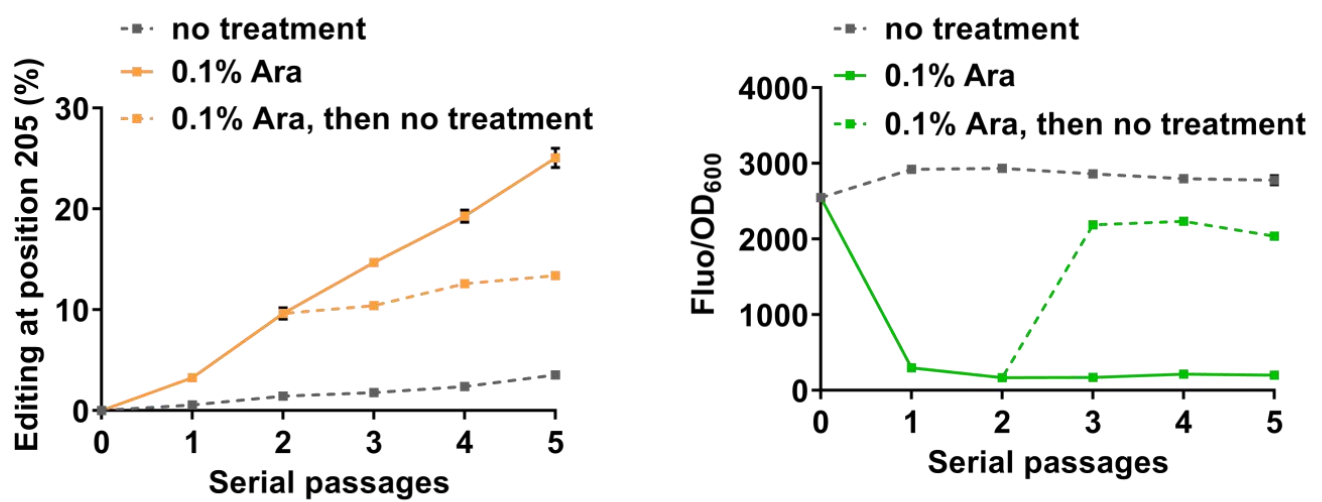

B
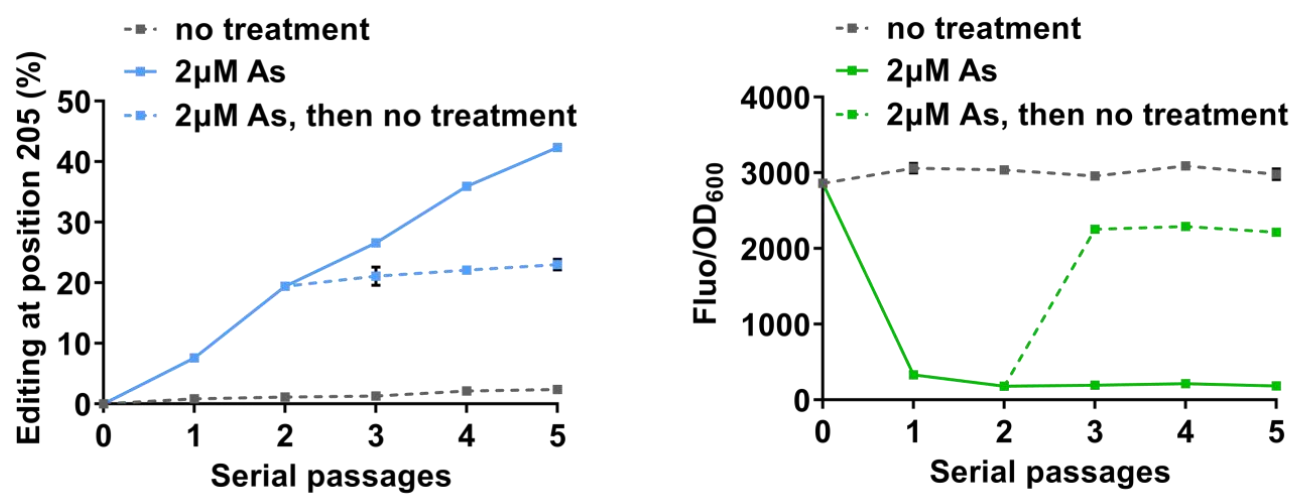

Figure S12. Characterization of the CRISPR-istop v2 system. (A) The rate of base editing at position 205 of the sfGFP gene in CRISPR-istop v2 reflects the schedule of exposure to the 0.1\%Ara (left) and the changing in the fluorescence of bacterial carrying arabinose-response elements in three cases (right). (B) The rate of base editing at position 205 of the sfGFP gene in CRISPR-istop v2 reflects the schedule of exposure to the $2 \mu \mathrm{M}$ As (left) and the changing in the fluorescence of bacterial carrying arsenic -response elements in three cases (right). Values and error bars are shown as mean $\pm \mathrm{SD}(\mathrm{n}=2)$. 

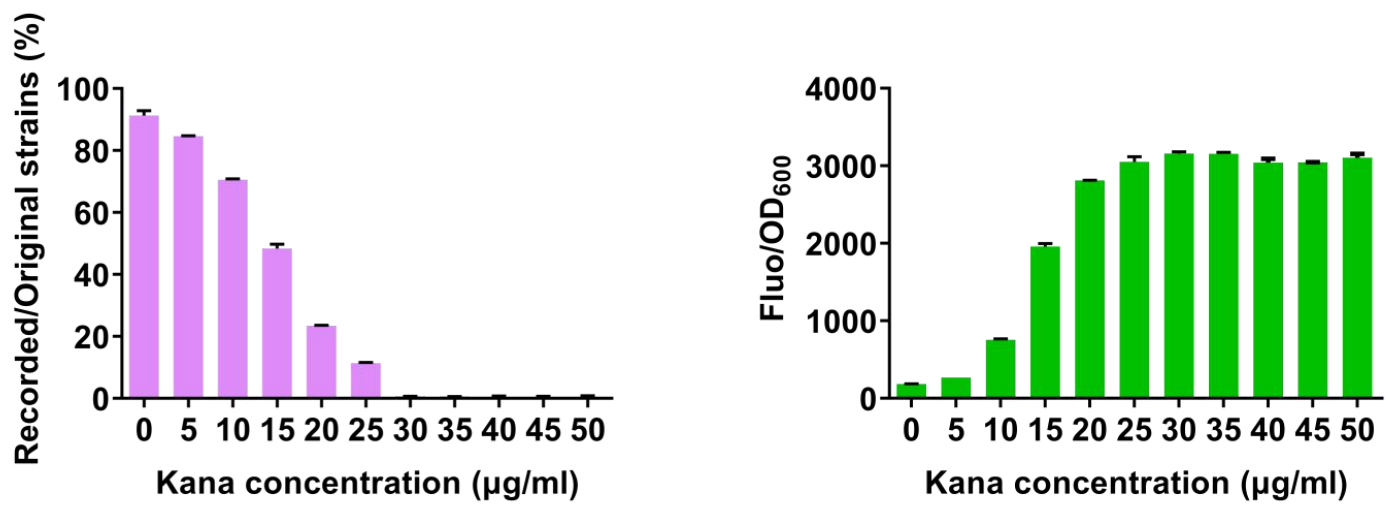

Figure S13. Kanamycin dose-dependently adjusted the ratio of the recorded bacterial to the original bacterial. Left: ratio of the recorded strains:original strains. Right: fluorescence at different ratios. Values and error bars are shown as mean $\pm \mathrm{SD}(\mathrm{n}=3)$. 
A

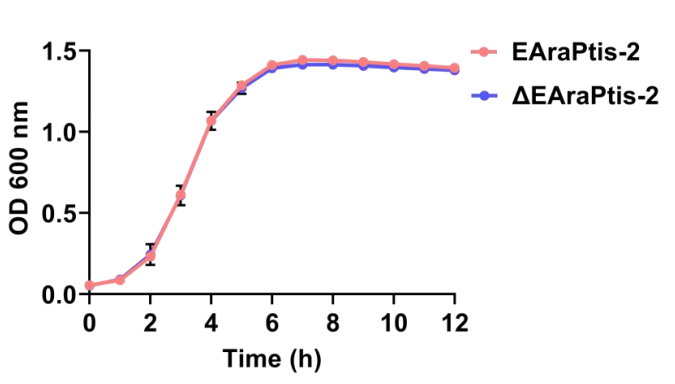

B

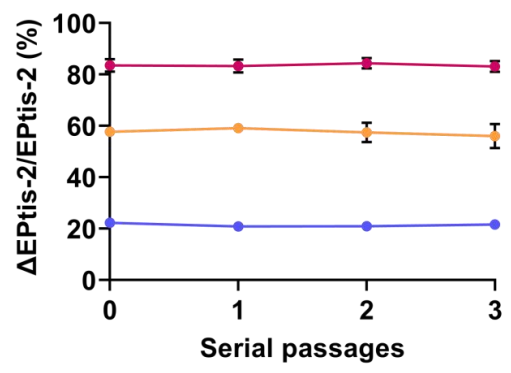

Figure S14. The effect of cellular fitness on CRISPR-istop v2 system. (A) Growth curves of EAraPtis-2 and $\Delta$ EAraPtis-2. (B) Continuously passages two strains of different proportions in the case of loss of writing plasmids, and the ratio change was measured. Values and error bars are shown as mean $\pm \operatorname{SD}(n=3)$. 


\section{Supplementary Tables}

Table S1. Summary of the strains used in this study

\begin{tabular}{cc}
\hline Strains name & Description \\
\hline EPt & MG1655::Ptac-sfGFP \\
EP1 & MG1655::Plac-sfGFP \\
EAraPti & MG1655::Plac-sfGFP/pWT-Ara-BE2-isg1 \\
EAraPli & MG1655::Ptac-sfGFP/pWT-Ara-BE2-ssg \\
EAraPts & MG1655::Plac-sfGFP/pWT-Ara-BE2-ssg \\
EAraPls & MG1655::Plac- $\Delta$ sfGFP/pWT-Ara-BE2-ssg \\
$\Delta$ EAraPls & MG1655::Ptac-sfGFP/pWT-Ara-BE2-isg1-ssg \\
EAraPtis & MG1655::Plac-sfGFP/pWT-Ara-BE2-isg1-ssg \\
EAraPlis & MG1655::Ptac-sfGFP/pWT-As-BE2-isg1-ssg \\
EAsPtis & MG1655::Ptac-sfGFP-KanR/pWT-Ara-BE2-isg2-ssg \\
EAraPtis-2 & MG1655::Ptac-sfGFP-KanR/pWT-As-BE2-isg2-ssg \\
EAsPtis-2 & MG1655::Ptac- $\Delta$ sfGFP- $\Delta$ \\
$\Delta$ EAraPtis-2 & KanR/pWT-As-BE2-isg2-ssg \\
& MG1655::Ptac-sfGFP-KanR \\
EPtis-2 & MG1655::Ptac- $\Delta$ sfGFP- $\Delta$ KanR \\
$\Delta$ EPtis-2 &
\end{tabular}


Table S2. Summary of plasmid constructs

\begin{tabular}{ccccc}
\hline Plasmid name & $\begin{array}{c}\text { Antibiotic } \\
\text { resistance }\end{array}$ & Promoter & Elements & Reference \\
\hline pWT021a & Strep & $\mathrm{P}_{\text {TetO }}$ & BE2 & {$[1]$} \\
pWT-Pj23119-isgRNA1 & Strep & $\mathrm{P}_{\text {TetO }}$ & BE2, isgRNA1 & This study \\
pWT-Ara-BE2-isg1 & Strep & $\mathrm{P}_{\mathrm{BAD}}$ & BE2, isgRNA1 & This study \\
pWT-Ara-BE2-ssg & Strep & $\mathrm{P}_{\mathrm{BAD}}$ & BE2,ssgRNA & This study \\
pWT-Ara-BE2-isg1-ssg & Strep & $\mathrm{P}_{\mathrm{BAD}}$ & BE2, isgRNA1, ssgRNA & This study \\
pWT-Ara-BE2-isg2 & Strep & $\mathrm{P}_{\mathrm{BAD}}$ & BE2, isgRNA2 & This study \\
pWT-Ara-BE2-isg2-ssg & Strep & $\mathrm{P}_{\mathrm{BAD}}$ & BE2, isgRNA2, ssgRNA & This study \\
ParsD-ABS-10 & Tet & $\mathrm{P}_{\text {ARS }}$ & sfGFP & {$[2]$} \\
pWT-arsR & Strep & $\mathrm{P}_{\mathrm{ARS}}$ & sfGFP & This study \\
pWT-msfGFP-KanR & Strep & $\mathrm{P}_{\text {tac }}$ & msfGFP, KanR & This study \\
pWT-m $\Delta$ sfGFP-KanR & Strep & $\mathrm{P}_{\text {tac }}$ & m $\Delta$ sfGFP, KanR & This study \\
pWT-As-BE2-isg1-ssg & Strep & $\mathrm{P}_{\mathrm{ARS}}$ & BE2, isgRNA1, ssgRNA & This study \\
pWT-As-BE2-isg2-ssg & Strep & $\mathrm{P}_{\text {ARS }}$ & BE2, isgRNA2, ssgRNA & This study \\
\hline
\end{tabular}


Table S3. Summary of the synthetic DNA fragments/primers used in this study

\begin{tabular}{|c|c|c|}
\hline $\begin{array}{c}\text { Synthetic DNA } \\
\text { fragment/Primers } \\
\text { name }\end{array}$ & Sequences $\left(5^{\prime}\right.$ to $\left.3^{\prime}\right)$ & Description \\
\hline Pj23119-isgRNA1 & $\begin{array}{l}\text { ctcgtgcatactgcgtatgatgagtcgaccgatgaaaa } \\
\text { cgtcatgctgctgacctccgacgcgeccgagtataaaccg } \\
\text { tgggetttggttatccaggatagcaacggtgaaaataagat } \\
\text { taaaatgttataaacggagccaatgtacgcaaaaaccccg } \\
\text { cttcagcggggttttttcgecaaaaaaaaccccgccctgtc } \\
\text { aggggcggggttttttttatgttgaaaatctccttctaaaaa } \\
\text { aagcaccgactcggtgccacttttcaagttgataacggac } \\
\text { tagccttattttaacttgctatttctagctctaaaactattctgg } \\
\text { tggaactggatggctagcattatacctaggactgagctag } \\
\text { ctgtcaaattgcgttgcgcacttaattaacggcactcctcag } \\
\text { caaatata }\end{array}$ & Plasmid construction \\
\hline H-APOBEC-F & atgtcttctgaaaccggtccg & Plasmid construction \\
\hline sgRNA-R & tatatttgctgaggagtgccgttaa & Plasmid construction \\
\hline $\mathrm{H}$-araC-F & $\begin{array}{c}\text { ctcattttcgccagatatcgacgtcttatgacaacttgacgg } \\
\text { ctacatcat }\end{array}$ & Plasmid construction \\
\hline $\begin{array}{l}\operatorname{araBAD}-\mathrm{APOBEC}- \\
\mathrm{R}\end{array}$ & $\begin{array}{c}\text { cggaccggtttcagaagacatttttataacctccttagagc } \\
\text { tcgaatt }\end{array}$ & Plasmid construction \\
\hline smR-F & ttaacggcactcctcagcaaatata & Plasmid construction \\
\hline H-T1 ter-R & $\begin{array}{c}\text { gacgtcgatatctggcgaaaatgagataaaacgaaaggc } \\
\text { ccagtc }\end{array}$ & Plasmid construction \\
\hline G-ssgRNA1-F & $\begin{array}{c}\text { gataacgagcaaagcactgagctagcattatacctaggac } \\
\text { tgagctagct }\end{array}$ & Plasmid construction \\
\hline G-ssgRNA1-R & $\begin{array}{c}\text { tcagtgctttgctcgttatcgttttagagctagaaatagcaag } \\
\text { ttaaaat }\end{array}$ & Plasmid construction \\
\hline sgRNA-F & ctcgtgcatactgcgtatgatgagt & Plasmid construction \\
\hline Pj23119-Linker-R & $\begin{array}{c}\text { aattaacagaaaattattttaaatttcctcttgacagctagctc } \\
\text { agtcctagg }\end{array}$ & Plasmid construction \\
\hline Pj23119-Linker-F & $\begin{array}{l}\text { gtcaagaggaaatttaaaataattttctgttaattaaaaaaaa } \\
\text { ccccgccetgtc }\end{array}$ & Plasmid construction \\
\hline G-isgRNA2-F & $\begin{array}{l}\text { tattctggtccaactctatggetagcattatacctaggactg } \\
\text { agctagct }\end{array}$ & Plasmid construction \\
\hline G-isgRNA2-R & $\begin{array}{c}\text { catagagttggaccagaatagttttagagctagaaatagca } \\
\text { agttaaaat }\end{array}$ & Plasmid construction \\
\hline
\end{tabular}




\begin{tabular}{|c|c|c|}
\hline$H-\operatorname{arsR}-\mathrm{F}$ & $\begin{array}{c}\text { ctcattttcgecagatatcgacgtcttaactgcaaatgttctt } \\
\text { actgtcccc }\end{array}$ & Plasmid construction \\
\hline H-GFP-issg-R & $\begin{array}{c}\text { gtttttgegtacattggetccgtctatttgtatagttcatccatg } \\
\text { ccatgtg }\end{array}$ & Plasmid construction \\
\hline GFP-issg-F & acggagccaatgtacgcaaaaac & Plasmid construction \\
\hline sg-arsR-T1-R & gacgtcgatatctggcgaaaatgag & Plasmid construction \\
\hline H-arsR-ZL-R & $\begin{array}{l}\text { ccggaccggtttcagaagacatatgtttttcctccttataaa } \\
\text { gttaatctttagttagt }\end{array}$ & Plasmid construction \\
\hline GFP-issg-R & gtttttgcgtacattggetccgt & Plasmid construction \\
\hline cx-istop-GF & cgaagaaggggttgaatcgc & Sequalizer analysis primers \\
\hline cx-istop-GR & caggatattgecgtcttctttaaag & Sequalizer analysis primers \\
\hline cx-dCas9-F & tcagctaggaggtgacagcg & Sequencing primers \\
\hline cx-SmR-R & caaataaacgccatgggcat & Sequencing primers \\
\hline cx-sfGFP-F & ggtgtatatggcgagcgcaat & Sequencing primers \\
\hline cx-KanR-R & ggctggtcttcatccacgcg & Sequencing primers \\
\hline STY-G-F & gccggaaggcgaagcgg & Genomic DNA knock-in primer \\
\hline H-GFP-pET-R & $\begin{array}{l}\text { accccgtagaaaagatcaaaggatcttcatcatttgtacag } \\
\text { ttcatccataccat }\end{array}$ & Genomic DNA knock-in primer \\
\hline H-pET-GFP-R & accccgtagaaaagatcaaaggat & Genomic DNA knock-in primer \\
\hline pET-KanR-F & gaagatcctttgatcttttctacggggt & Genomic DNA knock-in primer \\
\hline XTY1-R & agcgccgtggcctgattcat & Genomic DNA knock-in primer \\
\hline
\end{tabular}


Table S4. Kanamycin inhibits mutant bacterial growth

\begin{tabular}{|c|c|c|c|c|}
\hline & Starting ratio & $+\mathrm{S}$ & $+\mathrm{K}$ & $+\mathrm{S}$ and $\mathrm{K}$ \\
\hline \multirow{2}{*}{$\begin{array}{c}\mathrm{T} / \mathrm{C} \text { at position } 205 \\
(\%)\end{array}$} & 69.2 & 83.5 & 0.2 & 0.1 \\
\cline { 2 - 5 } & 46.3 & 69.9 & 0.2 & 0.6 \\
\cline { 2 - 5 } & 30.8 & 50.1 & 0.3 & 0.2 \\
\hline
\end{tabular}

+ S: add streptomycin. + K: add kanamycin

\section{REFERENCE:}

(1) Tang, W., and Liu, D. R. (2018) Rewritable multi-event analog recording in bacterial and mammalian cells. Science 360 , eaap8992.

(2) Chen, S. Y., Wei, W., Yin, B. C., Tong, Y., Lu, J., and Ye, B. C. (2019) Development of a highly sensitive whole-cell biosensor for arsenite detection through engineered promoter modifications. ACS Synth. Biol. 8, 2295-2302. 